Pacific

Journal of

Mathematics

GEOMETRY OF THE UNIT BALL AND REPRESENTATION THEORY FOR OPERATOR

ALGEBRAS

Elias G. Katsoulis 


\title{
GEOMETRY OF THE UNIT BALL AND REPRESENTATION THEORY FOR OPERATOR ALGEBRAS
}

\author{
Elias G. KATSOULiS
}

\begin{abstract}
We investigate the relationship between the facial structure of the unit ball of an operator algebra $\mathcal{A}$ and its algebraic structure, including the hereditary subalgebras and the socle of $\mathcal{A}$. Many questions about the facial structure of $\mathcal{A}$ are studied with the aid of representation theory. For that purpose we establish the existence of reduced atomic type representations for certain nonselfadjoint operator algebras. Our results are applicable to $\mathrm{C}^{*}$-algebras, strongly maximal TAF algebras, free semigroup algebras and various semicrossed products.
\end{abstract}

The study of geometric problems in operator algebra theory goes back to the beginnings of the subject. The theory of Gelfand-Naimark and Segal identified the extreme points of the state space of a $\mathrm{C}^{*}$-algebra as functionals (pure states) that produce irreducible representations under the GNS machinery. Kadison's characterization of the isometric linear maps between $\mathrm{C}^{*}$-algebras $[29]$ depended heavily on the identification of the extreme points for the unit ball. Crucial information about the algebraic structure of a $\mathrm{C}^{*}$ algebra is encoded in the geometry of its unit ball. The ideal structure of the algebra coincides with the M-structure [4] and the density of the invertibles is reflected in the richness of the convex hull of the unitary operators [45].

A subset $\mathcal{F}$ of a convex set $\mathcal{K}$ is said to be a face of $\mathcal{K}$ if it is convex and has the property that, if an interior point of a line segment in $\mathcal{K}$ belongs to $\mathcal{F}$, the entire line segment belongs to $\mathcal{F}$. The extreme points of a convex set, together with the empty set, form the trivial faces of $\mathcal{K}$. A face $\mathcal{F}$ is said to be finite-dimensional if the (real) linear space generated by $\mathcal{F}$ is finite-dimensional. If $\mathcal{K}$ is contained in a normed linear space, then $\mathcal{F}$ is said to be compact if its norm closure is a norm compact set. A comprehensive study of the facial structure for the unit ball of a $\mathrm{C}^{*}$-algebra was conducted by Akemann and Pedersen [2], following related work by Edwards and Ruttimann $[\mathbf{2 2}, \mathbf{2 3}]$. Beyond selfadjoint operator algebras, there has not been a systematic work addressing the nontrivial faces of the unit ball.

In this paper we begin a study of the nontrivial compact faces of the unit ball of an arbitrary operator algebra. (All operator algebras are assumed to be norm closed and contain the identity operator.) The existence of such 
faces has a significant impact on the structure of the algebra. In Theorem 1.6 we show that if the unit ball of an operator algebra $\mathcal{A}$ has a nontrivial compact face $\mathcal{F}$, then $\mathrm{S}(\mathcal{F})$, and therefore $\mathcal{A}$, contains a nonscalar operator $A$ whose spectrum has at most one limit point. (Here $\mathrm{S}(\mathcal{F})$ denotes the unique real subspace of $\mathcal{A}$ that is a translate of the affine hull of $\mathcal{F}$.) For a finite-dimensional face $\mathcal{F}$ we can offer a more definitive result. Theorem 1.8 shows that $\mathrm{S}(\mathcal{F})$ is a (real) finite-dimensional hereditary subalgebra of $\mathcal{A}$ consisting of multiples of elements with finite geometric rank. Moreover, if $\mathcal{A}$ happens to be semisimple then $\mathrm{S}(\mathcal{F})$ is contained in the socle of $\mathcal{A}$; this makes an important connection between the facial structure of the unit ball and the general theory of Banach Algebras. As a consequence, if the unit ball of a semisimple operator algebra $\mathcal{A}$ contains a nontrivial finitedimensional face then $\mathcal{A}$ contains a minimal idempotent (Corollary 1.10). We also relate the existence of nontrivial compact faces with the concept of geometric compactness, which was first introduced by Anoussis and the author in $[\mathbf{5}, \mathbf{6}]$. Theorem 1.3 shows that the existence of nontrivial compact faces implies the existence of geometrically compact elements. Actually, we observe that just the presence of nonzero geometrically compact elements suffices for the existence of nonscalars with discrete spectrum.

The general results of the first section are complemented with several applications. In the second section of the paper we investigate the facial structure of various operator algebras with the aid of representation theory. Motivated by our earlier work in [5], we introduce the class of operator semisimple algebras; these are algebras that can be isometrically represented as a strongly dense subalgebra of the diagonal algebra $\bigoplus_{a \in \mathbb{A}} \mathcal{B}\left(\mathcal{H}_{a}\right)$. We prove that for an operator semisimple algebra $\mathcal{A}$ the existence of nontrivial compact faces, the existence of nonzero geometrically compact elements and the existence of atoms are all equivalent conditions. Moreover, we relate the concept of geometric compactness to the representation theory of $\mathcal{A}$. In Theorem 2.4 we show that an element $A \in \mathcal{A}_{1}$ is geometrically compact if and only if there exists an isometric representation $\varphi$ of $\mathcal{A}$ such that $\varphi(A)$ is a compact operator. This generalizes our earlier selfadjoint work [5] to the nonselfadjoint setting.

The rest of the second section is occupied with identifying various classes of operator semisimple algebras. Clearly, any operator algebra containing the compacts acts irreducibly on the Hilbert space and hence is operator semisimple. By an old result of Gardner, all $\mathrm{C}^{*}$-algebras are also operator semisimple. Therefore, the unit ball of a unital $\mathrm{C}^{*}$-algebra $\mathcal{A}$ has nontrivial compact faces if and only if it has finite-dimensional faces if and only if $\mathcal{A}$ has an atom. This result was implicit in [5].

It turns out that the concept of operator semisimplicity is also applicable to TAF algebras, a class of nonselfadjoint algebras that has received a great deal of attention in recent years; cf. Power's monograph [44]. Here 
we use the representation theory of Davidson and the author [13]. In [13] we characterized the operator primitive TAF algebras as the semisimple ones whose enveloping $\mathrm{C}^{*}$-algebra is primitive. Here we add to this result and in Theorem 2.6 we show that all semisimple TAF algebras are operator semisimple. As an immediate corollary of our theory, the unit balls of the familiar standard, alternation and $A(\mathbb{Q}, \nu)$ algebras do not contain any nontrivial compact faces.

The list of operator semisimple algebras also includes various function algebras. Indeed, in Theorem 2.9 we show that if the unitary functions in a uniform algebra $\mathcal{A}$ separate points, $\mathcal{A}$ is operator semisimple. In particular, $\mathrm{H}^{\infty}$ and the disc algebra $\mathrm{A}(\mathbb{D})$ are operator semisimple. The methods of the second section are also applicable to semicrossed products of the form $\mathrm{C}(\mathbb{T}) \times{ }_{\alpha} \mathbb{Z}^{+}$, where $\alpha$ is an irrational rotation of the circle $\mathbb{T}$. Indeed, in [14] it is shown that such algebras are operator primitive. Therefore, the unit ball of such an algebra does not contain any nontrivial compact faces.

In the third section, we study the presence of compact faces in the unit ball of a free semigroup algebra. In [15, Theorem 4.5] it is shown that every operator in the open unit ball of a free semigroup algebra $\mathcal{A}$ is a mean of isometries from $\mathcal{A}$. This generalizes a classical result of Marshall and shows that there is an abundance of extreme points in the unit ball of these algebras. Theorem 3.1 shows now that, once again, the unit ball of a free semigroup algebra $\mathcal{A}$ contains nontrivial compact faces if and only if $\mathcal{A}$ has atoms. In particular, the unit ball of the "noncommutative Toeplitz algebra" $\mathcal{L}_{n}$ has no nontrivial compact faces, a result that seems to be new even for $\mathrm{H}^{\infty}$. We note that the representation techniques of the second section are not applicable here since a free semigroup algebra may not be semisimple. Instead we use the spectral properties of $\mathcal{L}_{n}$ together with the structure theorem of Davidson, Katsoulis and Pitts [15]. Similar spectral considerations also show that the unit ball of $\mathrm{A}(\mathbb{D}) \times_{\alpha} \mathbb{Z}^{+}$and $\mathrm{H}^{\infty} \times_{\alpha} \mathbb{Z}^{+}$ do not contain any nontrivial finite-dimensional faces. These algebras were studied in $[\mathbf{2 7}, \mathbf{2 8}]$.

The last section of the paper contains several remarks and observations, including a generalization of Kadison's characterization for the extreme points of the unit ball of a $\mathrm{C}^{*}$-algebra.

\section{Structure for the faces of the unit ball}

If $\mathcal{K} \subseteq \mathcal{X}$ is a convex subset of a complex normed space $\mathcal{X}$, then $[\mathcal{K}]_{\mathbb{R}}$ denotes the real subspace of $\mathcal{X}$ generated by $\mathcal{K}$ :

$$
[\mathcal{K}]_{\mathbb{R}} \equiv\left\{\sum_{i=1}^{n} \lambda_{i} x_{i} \mid \lambda_{i} \in \mathbb{R}, x_{i} \in \mathcal{K}, 1 \leq i \leq n, n \in \mathbb{N}\right\}
$$


(The complex subspace generated by $\mathcal{K}$ will be denoted as $[\mathcal{K}]$.) For any $x \in \mathcal{K}$, the subspace $[x-\mathcal{K}]_{\mathbb{R}}$ does not depend on the choice of $x \in \mathcal{K}$ and is denoted as $\mathrm{S}(\mathcal{K})$. The translation of $\mathrm{S}(\mathcal{K})$ by any element of $\mathcal{K}$ equals the affine hull of $\mathcal{K}$.

If $x$ and $y$ belong to $\mathcal{K}$, the line segment joining $x$ and $y$ is denoted by $[x, y]$. Thus

$$
[x, y]=\{\lambda x+(1-\lambda) y \mid \lambda \in[0,1]\} .
$$

If $x$ and $y$ are in $\mathcal{K}$ and $x \neq y$, then an element $v \in[x, y]$ is said to be an internal point of $[x, y]$ if $v \neq x$ and $v \neq y$. Given $v \in \mathcal{K}$, we write $\mathcal{F}(\mathcal{K}, v)$ for the union of all line segments in $\mathcal{K}$ that contain $v$ as an internal point, provided that $v$ is not an extreme point of $\mathcal{K}$. Otherwise, $\mathcal{F}(\mathcal{K}, v)=\{v\}$. If $\mathcal{K}$ is the unit ball of $\mathcal{X}$, then $\mathcal{F}(\mathcal{K}, v)$ is simply denoted as $\mathcal{F}(v)$. It is an important fact in elementary convexity theory that for each $v \in \mathcal{K}$, the set $\mathcal{F}(\mathcal{K}, v)$ is a face of $\mathcal{K}$ that is minimal with the property of containing $v$. (The proof of this is an entertaining exercise in plane geometry; or see $[\mathbf{1}$, Theorem 1.2] for a detailed proof.)

If $\mathcal{S}$ is a nonempty subset of the unit ball of $\mathcal{X}$, then the contractive perturbations of $\mathcal{S}$ are defined as

$$
\operatorname{cp}(\mathcal{S})=\{x \in \mathcal{X} \mid\|x \pm s\| \leq 1 \text { for all } s \in \mathcal{S}\} .
$$

It is clear that $\mathcal{S}_{1} \subseteq \mathcal{S}_{2}$ implies $\operatorname{cp}\left(\mathcal{S}_{1}\right) \supseteq \operatorname{cp}\left(\mathcal{S}_{2}\right)$. Also, an element of the unit ball of $\mathcal{X}$ is an extreme point if and only if $\operatorname{cp}(\{x\})=\{0\}$.

The following result relates contractive perturbations with the facial structure of the unit ball:

Lemma 1.1. Let $\mathcal{F}$ be a face of the unit ball of a normed space $\mathcal{X}$. If $x \in \mathcal{F}$, then,

$$
\operatorname{cp}(\{x\}) \subseteq \frac{1}{2}(\mathcal{F}-\mathcal{F}) .
$$

Proof. Let $m \in \operatorname{cp}(\{x\})$. Then $x=\frac{1}{2}((x+m)+(x-m))$, and therefore $x \pm m \in \mathcal{F}$. Hence $m=\frac{1}{2}(x+m-(x-m))$ belongs to $\frac{1}{2}(\mathcal{F}-\mathcal{F})$ and this proves the lemma.

We now compute $\mathrm{S}(\mathcal{F}(x))$ in terms of contractive perturbations for $x$.

Lemma 1.2. Let $\mathcal{X}$ be a normed space and let $x \in \mathcal{X}_{1}$. Then

$$
[x-\mathcal{F}(x)]_{\mathbb{R}}=[\operatorname{cp}(\{x\})]_{\mathbb{R}} .
$$

Proof. Assume $x+m \in \mathcal{F}(x)$. There exists $\lambda>0$ such that $x \pm \lambda m \in \mathcal{F}(x)$, so $\lambda m \in \operatorname{cp}(\{x\})$. Conversely, if $m \in \operatorname{cp}(\{x\})$, then $[x-m, x+m] \subseteq \mathcal{X}_{1}$ and so by the definition of $\mathcal{F}(x)$ we have that $x \pm m \in \mathcal{F}(x)$.

One may define contractive perturbations of higher-order by using the recursive formula $\mathrm{cp}^{(n+1)}(\mathcal{S})=\mathrm{cp}\left(\mathrm{cp}^{(n)}(\mathcal{S})\right), n \in \mathbb{N}$. These higher-order contractive perturbations satisfy the Galois duality $\mathrm{cp}^{(n+2)}(\mathcal{S})=\mathrm{cp}^{(n)}(\mathcal{S})$, 
$n \in \mathbb{N}$. The second contractive perturbations were introduced by Anoussis and the author in $[\mathbf{5}, \mathbf{6}]$. In [5] we defined a contraction $x$ in a normed space space $\mathcal{X}$ to be geometrically compact if $\mathrm{cp}^{(2)}(\{x\})$ is norm compact. If $\mathrm{cp}^{(2)}(\{x\})$ happens to span a finite-dimensional subspace of $\mathcal{X}$, then $x$ is said to have finite geometric rank. In [5, Theorem 2.2] we proved that a nonzero element $A$ of a $\mathrm{C}^{*}$-algebra $\mathcal{A}$ is geometrically compact (resp. has finite geometric rank) if and only if there exists a faithful representation $\varphi$ of $\mathcal{A}$ such that $\varphi(A)$ is a compact operator (resp. $\varphi(A)$ is a finite rank operator).

Theorem 1.3. Let $\mathcal{X}$ be a normed space and assume that the unit ball of $\mathcal{X}$ has a nontrivial compact face $\mathcal{F}$. Then $\mathrm{S}(\mathcal{F})$ contains a nonzero geometrically compact element.

Proof. Let $x_{1}, x_{2}$ be distinct elements of $\mathcal{F}$ and let $x=\frac{1}{2}\left(x_{1}+x_{2}\right)$. Since $x$ is not an extreme point, $\operatorname{cp}(\{x\})$ contains a nonzero element, say $m$. Then $\{m\} \subseteq \operatorname{cp}(\{x\})$ and so

$$
\mathrm{cp}^{(2)}(\{m\}) \subseteq \mathrm{cp}^{(3)}(\{x\})=\operatorname{cp}(\{x\}) .
$$

By Lemma 1.1, $\mathrm{cp}^{(2)}(m)$ is contained in $\frac{1}{2}(\mathcal{F}-\mathcal{F})$, which is a norm compact set; thus $m$ is geometrically compact. Hence $\operatorname{cp}(\{x\})$ contains nonzero geometrically compact elements and by Lemma 1.2 the same is true for $[x-\mathcal{F}(x)]_{\mathbb{R}} \subseteq[x-\mathcal{F}]_{\mathbb{R}}$.

It is instructive to observe that some geometrically compact elements may be located outside translates of affine hulls for compact faces. Actually, there exists a Banach space $\mathcal{X}$ containing elements with finite geometric rank but its unit ball has no compact faces. Indeed, by [5, Theorem 2.2], $c_{0}$ contains an abundance of elements with finite geometric rank. However, given any element $x$ in the unit ball of $c_{0}$, it is easy to see that $\mathcal{F}(x)$ does not have a compact closure and so the unit ball of $c_{0}$ has no compact faces.

The following mild generalization of [5, Proposition 1.2] is necessary for deriving Theorem 1.8. Compare with [34, Theorem 3] and [46, Theorem 2], where the calculations below originate.

Proposition 1.4. Let $\mathcal{A}$ be an operator algebra, let $\emptyset \neq \mathcal{S} \subseteq \mathcal{A}_{1}$ and assume that $S_{1}, S_{2} \in \mathcal{S}$. If $X \in \mathcal{A}$ satisfies $\|X\| \leq 1 / 2$, then $S_{1} X S_{2} \in \mathrm{cp}^{(2)}(\mathcal{S})$.

Proof. Let $B \in \operatorname{cp}(\mathcal{S})$. Since $\left\|S_{i} \pm B\right\| \leq 1$ we have

$$
\begin{aligned}
& S_{i}^{*} S_{i}+B^{*} B-S_{i}^{*} B-B^{*} S_{i} \leq I, \\
& S_{i}^{*} S_{i}+B^{*} B+S_{i}^{*} B+B^{*} S_{i} \leq I,
\end{aligned}
$$

so $S_{i}^{*} S_{i} \leq I-B^{*} B$. Douglas' majorization theorem implies the existence of a contraction $Q_{i}$ such that $S_{i}=Q_{i}\left(I-B^{*} B\right)^{1 / 2}$. A similar argument shows 
that $S_{i}=\left(I-B B^{*}\right)^{1 / 2} P_{i}$ for some contraction $P_{i}$. Hence,

$$
\begin{aligned}
S_{1} X S_{2} & =\left(I-B B^{*}\right)^{1 / 2} P_{1} X Q_{2}\left(I-B^{*} B\right)^{1 / 2}, \\
& =\left(I-\left|B^{*}\right|\right)^{1 / 2} Y(I-|B|)^{1 / 2},
\end{aligned}
$$

where $Y=\left(I+\left|B^{*}\right|\right)^{1 / 2} P_{1} X Q_{2}(I+|B|)^{1 / 2}$; thus $\|Y\| \leq 1$. The Heinz-Kato inequality [32] now asserts that for any vectors $e, f \in \mathcal{H}$ we have

$$
|\langle B e, f\rangle| \leq\left\||B|^{1 / 2} e\right\|\left\|\left|B^{*}\right|^{1 / 2} f\right\| \text {. }
$$

Combining (1) and (2), we obtain $\left\|B \pm S_{1} X S_{2}\right\| \leq 1$ and so $S_{1} X S_{2} \in$ $\mathrm{cp}^{(2)}(\mathcal{S})$, as desired.

Corollary 1.5. Let $\mathcal{A}$ be an operator algebra, $A \in \mathcal{A}_{1}$ and assume that $C_{1}, C_{2} \in \operatorname{cp}(\{A\})$. If $X \in \mathcal{A}$ satisfies $\|X\| \leq 1 / 2$, then $C_{1} X C_{2} \in \operatorname{cp}(\{A\})$.

Proof. Apply Proposition 1.4 with $\mathcal{S}=\operatorname{cp}(\{A\})$. Then

$$
C_{1} X C_{2} \in \mathrm{cp}^{(3)}(\{A\})=\operatorname{cp}(\{A\}) .
$$

If $a$ is an element of a Banach algebra $\mathfrak{A}$, let $\sigma_{\mathfrak{A}}(a)$ denote the spectrum of $a$ as an element of $\mathfrak{A}$. The left multiplier $L_{a}$ is defined as $L_{a} b=a b, b \in \mathfrak{A}$. The collection $\mathrm{M}_{l}(\mathfrak{A})$ of all left multipliers on $\mathfrak{A}$ is isometrically isomorphic as an algebra to $\mathfrak{A}$. Therefore, the map $a \longrightarrow L_{a}$ is spectrum preserving, i.e., $\sigma_{\mathfrak{A}}(a)=\sigma_{\mathrm{M}_{l}(\mathfrak{A})}\left(L_{a}\right)$.

Theorem 1.6. Let $\mathcal{A}$ be an operator algebra, let $\mathcal{F}$ be a nontrivial face of its unit ball and let $\mathrm{S}(\mathcal{F})$ be the unique real subspace of $\mathcal{A}$ that is a translate of the affine hull of $\mathcal{F}$. Then the following three conditions are successively weaker:

(i) $\mathcal{F}$ is a compact face.

(ii) $\mathrm{S}(\mathcal{F})$ contains a nonzero geometrically compact element.

(iii) $\mathrm{S}(\mathcal{F})$ contains a nonscalar operator $A$ whose spectrum has at most one limit point.

If $\mathcal{A}$ is commutative and semisimple, then condition (ii) also implies:

(iii') $\mathcal{A}$ contains a minimal idempotent, i.e., a nonzero idempotent $Q$ such that $Q \mathcal{A} Q=\mathbb{C} Q$.

Proof. (i) $\Rightarrow$ (ii): Theorem 1.3 shows that this is valid for any normed space.

(ii) $\Rightarrow$ (iii): Assume that $\mathrm{S}(\mathcal{F})$ contains such an element $A$, so the norm closure of $\mathrm{cp}^{(2)}(\{A\})$ is a nonzero compact set. Proposition 1.4 shows that

$$
A \mathcal{A}_{1 / 2} A \subseteq \mathrm{cp}^{(2)}(\{A\}),
$$

so the norm closure of $A \mathcal{A}_{1} A$ is norm compact.

Consider the elementary operator $L_{A^{2}}$ acting on the operator algebra $\mathfrak{A}$ generated by the polynomials of $A$. Since the closure of $A \mathcal{A}_{1} A$ is norm compact, the norm closure of $A^{2} \mathfrak{A}_{1}$ is also compact, so the operator $L_{A^{2}}$ 
is a compact operator on $\mathfrak{A}$. According to the Riesz theory for compact operators, $\sigma_{\mathcal{B}(\mathfrak{A})}\left(L_{A^{2}}\right)$ is a countable set with 0 as its only limit point (see Theorem VII.7.1 in [11]). Clearly the same is true for $\sigma_{\mathcal{B}(\mathfrak{A})}\left(L_{A}\right)$. Since $\mathrm{M}_{l}(\mathfrak{A}) \subseteq \mathcal{B}(\mathfrak{A})$, Theorem VII.5.4 in [11] shows that $\sigma_{\mathcal{B}(\mathfrak{A})}\left(L_{A}\right)$ and $\sigma_{\mathrm{M}_{l}(\mathfrak{A})}\left(L_{A}\right)$ differ only by holes, and so are equal. Hence, $\sigma_{\mathrm{M}_{l}(\mathfrak{A})}\left(L_{A}\right)$ is countable with one limit point. Our remarks above show that the same is true for $\sigma_{\mathfrak{A}}(A)$. Another application of [11, Theorem VII.5.4] for the Banach algebras $\mathfrak{A} \subseteq \mathcal{B}(\mathcal{H})$ shows that $\sigma_{\mathcal{B}(\mathcal{H})}(A)$ has at most one limit point, as desired.

(ii) $\Rightarrow$ (iii'): Arguing as above, let $A \in \mathcal{A}$, so that the closure of $A \mathcal{A}_{1} A$ is norm compact and so, by commutativity, the norm closure of $A^{2} \mathcal{A}_{1}$ is a compact set. Therefore, the left multiplier $L_{A^{2}}$ is a compact operator on $\mathcal{A}$. Since $\mathcal{A}$ is semisimple, the left multiplier algebra $\mathrm{M}_{l}(\mathcal{A})$ is also semisimple. Hence $L_{A^{2}}$ is a nonquasinilpotent compact operator. Let $\lambda$ be a nonzero eigenvalue of $L_{A}$ and let $E(\lambda)$ be the corresponding Riesz idempotent (see VII.6.9 in [11]). Since $L_{A} \in \mathrm{M}_{l}(\mathcal{A})$, we have $E(\lambda) \in \mathrm{M}_{l}(\mathcal{A})$, so there exists an idempotent $Q \in \mathcal{A}$ such that $E(\lambda)=L_{Q}$. By [11, Corollary VII.7.8] the idempotent $E(\lambda)=L_{Q}$ has finite-dimensional range, i.e., $Q \mathcal{A}$ is finite-dimensional. By [38, Proposition 4.3.12], $Q \mathcal{A}$ is semisimple, so by the Wedderburn-Artin Theorem, $Q \mathcal{A}$ is isomorphic to a direct sum of full matrix algebras. The existence of the minimal idempotent in $\mathcal{A}$ now follows.

Corollary 1.7. The unit ball of $\mathrm{H}^{\infty}$ has no compact faces apart from singletons.

A (real or complex) subalgebra $\mathcal{B}$ of an operator algebra $\mathcal{A}$ is said to be hereditary if $B_{1}, B_{2} \in \mathcal{B}$ implies $B_{1} \mathcal{A} B_{2} \subseteq \mathcal{B}$. For complex selfadjoint subalgebras of $\mathrm{C}^{*}$-algebras this definition coincides with the familiar definition of a hereditary subalgebra, as it appears in $[36,40]$. It is easy to see that if $\mathcal{B}$ is a real hereditary subalgebra of $\mathcal{A}$, then $[B]$ is a complex hereditary subalgebra of $\mathcal{A}$.

Theorem 1.8. Let $\mathcal{A}$ be an operator algebra, let $\mathcal{F}$ be a finite-dimensional face of the unit ball of $\mathcal{A}$ and let $\mathrm{S}(\mathcal{F})$ be the unique real subspace of $\mathcal{A}$ that is a translate of the affine hull of $\mathcal{F}$. Then $\mathrm{S}(\mathcal{F})$ is a finite-dimensional hereditary subalgebra of $\mathcal{A}$ consisting of multiples of elements with finite geometric rank. Moreover,

$$
\mathcal{F}=(A+\mathrm{S}(\mathcal{F})) \cap \mathcal{A}_{1}
$$

for any $A \in \mathcal{F}$.

Proof. If $A, B \in \mathcal{F}$, then $[A-\mathcal{F}]_{\mathbb{R}}=[B-\mathcal{F}]_{\mathbb{R}}$ and so

$$
A+[A-\mathcal{F}]_{\mathbb{R}}=B+(A-B)+[B-\mathcal{F}]_{\mathbb{R}}=B+[B-\mathcal{F}]_{\mathbb{R}} .
$$


Therefore it suffices to prove (3) for a specific $A \in \mathcal{F}$. Since $\mathcal{F}$ is a finitedimensional convex set, it has nonempty relative interior. Therefore, there exists $A \in \mathcal{F}$ and $\epsilon>0$ such that $B \in \mathcal{F}$ implies

$$
[A-\epsilon(B-A), B] \subseteq \mathcal{F} .
$$

We claim that $\mathcal{F}=\mathcal{F}(A)$. Indeed, $\mathcal{F}(A) \subseteq \mathcal{F}$. Conversely, let $B \in \mathcal{F}$. The definition of $\mathcal{F}(A)$ and (4) imply that

$$
[A-\epsilon(B-A), B] \subseteq \mathcal{F}(A),
$$

and so $B \in \mathcal{F}(A)$, which proves the claim.

Since $\mathcal{F}=\mathcal{F}(A)$, Lemma 1.2 shows that

$$
\mathrm{S}(\mathcal{F})=[A-\mathcal{F}]_{\mathbb{R}}=[\operatorname{cp}(A)]_{\mathbb{R}} .
$$

By Corollary 1.5, $\mathrm{S}(\mathcal{F})$ is a finite-dimensional hereditary subalgebra of $\mathcal{A}$.

Since $\operatorname{cp}(\{A\})$ is convex and $\operatorname{cp}(\{A\})=-\operatorname{cp}(\{A\})$, a moment's reflection shows that $[\operatorname{cp}(\{A\})]_{\mathbb{R}}$ consists of multiples of $\operatorname{cp}(\{A\})$. Hence $\mathrm{S}(\mathcal{F})$ consists of multiples of elements in $\operatorname{cp}(\{A\})$. However, if $X \in \operatorname{cp}(\{A\})$, then

$$
\mathrm{cp}^{(2)}(\{X\}) \subseteq \mathrm{cp}^{(3)}(\{A\})=\mathrm{cp}(\{A\}) .
$$

Therefore $X$ has finite geometric rank and so $\mathrm{S}(\mathcal{F})$ consists of multiples of elements with finite geometric rank.

It remains to verify (3). Let $B \in \mathrm{S}(\mathcal{F})$ be such that $\|A+B\|=1$. Then there exists $\lambda>0$ such that $\lambda B \in \operatorname{cp}(A)$, and so $\|A-\lambda B\| \leq 1$. Hence $[A-\lambda B, A+B] \subseteq \mathcal{A}_{1}$. Since $A$ is contained in the interior of the line segment, we conclude that $A+B \in \mathcal{F}$, as desired.

Recall that the socle of a semisimple Banach algebra $\mathcal{A}$ is defined as the sum of all minimal left ideals of $\mathcal{A}$. It coincides with the sum of all minimal right ideals [38, Proposition 8.2.8] and therefore it is a (not necessarily closed) two-sided ideal of $\mathcal{A}$. The study of the socle has been a central theme in the theory of Banach algebras. Our next result shows that the socle is also important for the geometry of the unit ball.

Corollary 1.9. Let $\mathcal{A}$ be an operator algebra and let $\mathcal{F}$ be a finite-dimensional face of the unit ball of $\mathcal{A}$. If $\mathcal{A}$ is semisimple then $\mathrm{S}(\mathcal{F})$ is contained in the socle of $\mathcal{A}$.

Proof. Let $A \in \mathrm{S}(\mathcal{F})$. By Theorem 1.8, $\mathrm{S}(\mathcal{F})$ is hereditary, so the operator $X \longrightarrow A X A, X \in \mathcal{A}$, has finite-dimensional range. By [3, Theorem 7.2], $A$ belongs to the socle of $\mathcal{A}$.

Corollary 1.10. Let $\mathcal{A}$ be a semisimple operator algebra. If the unit ball of $\mathcal{A}$ has a nontrivial finite-dimensional face then $\mathcal{A}$ contains a minimal idempotent.

Proof. The socle of a semisimple Banach algebra $\mathcal{A}$ is generated by the set of minimal idempotents of $\mathcal{A}$ [38, Proposition 8.2.8]. 
In particular, the unit ball of a simple operator algebra has no finitedimensional faces apart from singletons.

\section{Representation theorems for operator algebras}

Recall that Theorem 1.8 asserts that if $\mathcal{F}$ is a finite-dimensional face of the unit ball of an operator algebra then $\mathrm{S}(\mathcal{F})$ consists of scalar multiples of elements with finite geometric rank. So far the elements with finite geometric rank have been characterized for two classes of operator algebras: nest algebras [6] and $\mathrm{C}^{*}$-algebras [5]. Using representation theory, we now characterize the elements with finite geometric rank and the geometrically compact elements for a variety of nonselfadjoint algebras.

Our selfadjoint work in [5] suggests the following definition. (Also compare with $[\mathbf{4 7}]$.)

Definition 2.1. An operator algebra $\mathcal{A}$ is said to be operator semisimple if there exists a family of Hilbert space representations $\left(\tau_{a}, \mathcal{H}_{a}\right)$ of $\mathcal{A}, a \in \mathbb{A}$, such that their direct sum $\tau=\bigoplus_{a \in \mathbb{A}} \tau_{a}$ is an isometric isomorphism of $\mathcal{A}$ that maps the $(1+\epsilon)$-ball of $\mathcal{A}$ onto a strongly dense subset of $\bigoplus_{a \in \mathbb{A}} \mathcal{B}\left(\mathcal{H}_{a}\right)_{1}$, for some $\epsilon>0$. The family $\left(\tau_{a}, \mathcal{H}_{a}\right), a \in \mathbb{A}$ is said to implement the operator semisimplicity.

By Lemma 2.1 in [25], each of the representations $\tau_{a}, a \in \mathbb{A}$, is algebraically irreducible. Since $\tau=\bigoplus_{a \in \mathbb{A}} \tau_{a}$ is faithful for $\mathcal{A}$ we conclude that the intersection of all kernels of algebraically irreducible representations for $\mathcal{A}$ equals zero, i.e., an operator semisimple algebra is indeed semisimple.

The next result provides additional information for the operator $A$ appearing in Theorem 1.6 (iii).

Lemma 2.2. Let $\mathcal{A}$ be an operator semisimple algebra and let $\left(\tau_{a}, \mathcal{H}_{a}\right)$, $a \in \mathbb{A}$, be the family of representations of $\mathcal{A}$ implementing the operator semisimplicity. If $A$ is a geometrically compact element of $\mathcal{A}$ then $\tau(A)$ is a compact operator.

Proof. Proposition 1.4 shows that the norm closure of $A \mathcal{A}_{1 / 2} A$ is contained in $\mathrm{cp}^{(2)}(\{A\})$, which is a norm compact set. Therefore, the norm closure of $\tau(A) \tau\left(\mathcal{A}_{1+\epsilon}\right) \tau(A)$ is also compact. However, the weak closure of $\tau\left(\mathcal{A}_{1+\epsilon}\right)$ contains $\mathcal{B}(\mathcal{H})_{1}$ and so the norm closure of

$$
\tau(A) \mathcal{B}(\mathcal{H})_{1} \tau(A)
$$

is norm compact.

We now prove that $\tau_{a}(A)$ is a compact operator, for any $a \in \mathbb{A}$. Let $e \in \mathcal{H}_{a}$ be such that $\tau_{a}(A)^{*} e \neq 0$ and let $\left\{f_{k}\right\}_{k=1}^{\infty}$ be an arbitrary sequence of unit vectors from $\mathcal{H}_{a}$. By what we saw the previous paragraph, the sequence $\left\{\tau_{a}(A)\left(e \otimes f_{k}\right) \tau_{a}(A)\right\}_{k=1}^{\infty}$ has a norm convergent subsequence. However,

$$
\tau_{a}(A)\left(e \otimes f_{k}\right) \tau_{a}(A)=\left(\tau_{a}(A)^{*} e\right) \otimes\left(\tau_{a}(A) f_{k}\right),
$$


so the sequence $\left\{\tau_{a}(A) f_{k}\right\}_{k=1}^{\infty}$ has a norm convergent subsequence. This proves that $\tau_{a}(A)$ is a compact operator.

It remains to show that $\tau(A)$ is a compact operator. Let $\mathbb{B}$ be the collection of all finite subsets of $\mathbb{A}$. For each $b \in \mathbb{B}$, let $T_{b}$ be the diagonal operator satisfying $\left.T_{b}\right|_{\mathcal{H}_{a}}=\tau_{a}(A)$, for all $a \in b$, and $\left.T_{b}\right|_{\mathcal{H}_{a}}=0$ otherwise. The previous paragraph shows that $T_{b}$ is a compact operator, for any $b \in \mathbb{B}$. It suffices to show that the net $\left\{T_{b}\right\}_{b \in \mathbb{B}}$ converges in norm to $\tau(A)$.

By way of contradiction assume that the net $\left\{T_{b}\right\}_{b \in \mathbb{B}}$ does not converge in norm to $\tau(A)$. This is easily seen to imply the existence of an $\epsilon>0$ and a sequence $\left\{a_{n}\right\}_{n \in \mathbb{N}} \subseteq \mathbb{A}$ such that $\left\|\tau_{a_{n}}(A)\right\| \geq \epsilon$, for all $n \in \mathbb{N}$. Therefore there exist unit vectors $f_{n} \in \mathcal{H}_{a_{n}}$ such that $\left\|\tau_{a_{n}}(A) f_{n}\right\| \geq \epsilon$, for all $n \in \mathbb{N}$. However, the sequence $\left\{f_{n}\right\}_{n \in \mathbb{N}}$ converges weakly to zero and so an argument similar to that of the second paragraph of the proof shows that the sequence $\left\|\tau_{a_{n}}(A) f_{n}\right\|$ converges to zero, a contradiction.

Lemma 2.3. Let $\mathcal{A}$ be an operator semisimple algebra, let $\left(\tau_{a}, \mathcal{H}_{a}\right), a \in \mathbb{A}$, be the family of representations of $\mathcal{A}$ implementing the operator semisimplicity and let $\tau=\bigoplus_{a \in \mathbb{A}} \tau_{a}$. Then the set of compact operators in $\tau(\mathcal{A})$ forms a $\mathrm{C}^{*}$-algebra.

Proof. Let $T=\bigoplus_{a \in \mathbb{A}} T_{a}$ be a compact operator in $\tau(\mathcal{A})$. Fix an $a_{0} \in \mathbb{A}$ and let $\left\{A_{i}\right\}_{i \in I}$ be a bounded net in $\tau(\mathcal{A})$ converging strongly to the operator that equals $T_{a_{0}}^{\star}$ on $\mathcal{H}_{a_{0}}$ and 0 everywhere else. Then $\left\{A_{i} T\right\}_{i \in I}$ converges in norm to a positive compact operator supported on $\mathcal{H}_{a_{0}}$. An application of the Spectral Theorem shows now that $\tau(\mathcal{A})$ contains a finite rank projection $P$ supported on $\mathcal{H}_{a_{0}}$.

We claim that $\tau(\mathcal{A})$ contains all rank one operators supported on $\mathcal{H}_{a_{0}}$. (This will imply that $\tau(\mathcal{A})$ contains all compact operators supported on $\mathcal{H}_{a_{0}}$ and in particular $T_{a_{0}}^{*}$.) Indeed, fix a unit vector $g \in P(\mathcal{H})$ and let $e \otimes f$ be any rank one operator supported on $\mathcal{H}_{a_{0}}$. Since $\tau$ implements an operator semisimplicity, there exists a bounded net $\left\{B_{i}\right\}_{i \in I}$ in $\tau(\mathcal{A})$ converging strongly to $g \otimes f$. Hence, the net $\left\{B_{i} P\right\}_{i \in I}$ converges in norm to $(g \otimes f) P=g \otimes f$ and so $g \otimes f \in \tau(\mathcal{A})$. Similarly, there exists a bounded net $\left\{C_{i}\right\}_{i \in I}$ in $\tau(\mathcal{A})$ converging strongly to $e \otimes g$ and so $\left\{C_{i}^{*}\right\}_{i \in I}$ converges weakly to $g \otimes e$. By $\left[\mathbf{1 1}\right.$, Corollary IX.5.2], there exists a net $\left\{D_{j}\right\}_{j \in J} \subseteq \tau(\mathcal{A})$ consisting of convex combinations from $\left\{C_{i}\right\}_{i \in I}$ and such that $\left\{D_{j}^{*}\right\}_{j \in J}$ converges strongly to $g \otimes e$. Hence, the net $\left\{D_{j}^{*} P\right\}_{j \in J}$ converges in norm to $(g \otimes e) P=g \otimes e$ and so $g \otimes e \in(\tau(\mathcal{A}))^{*}$. Hence, $e \otimes g \in \tau(\mathcal{A})$ and so

$$
e \otimes f=(g \otimes f)(e \otimes g) \in \tau(\mathcal{A}),
$$

as desired.

Finally, an approximation argument similar to that of the last paragraphs of the proof of Lemma 2.1, combined with the above claim, shows that $T^{*} \in \tau(\mathcal{A})$. 
The next result clarifies the nature of the geometrically compact elements in operator semisimple algebras and generalizes the main result in [5].

Theorem 2.4. Let $\mathcal{A}$ be an operator semisimple algebra and let $A \in \mathcal{A}$. Then $A$ is geometrically compact if and only if there exists an isometric representation $\varphi$ of $\mathcal{A}$ such that $\varphi(A)$ is a compact operator.

Proof. If $\mathcal{A}$ is geometrically compact then Lemma 2.2 shows that $\tau(A)$ is a compact operator.

Conversely, assume that there exists an isometric representation $\varphi$ of $\mathcal{A}$ such that $\varphi(A)$ is a compact operator. Then $\varphi(A) \varphi\left(\mathcal{A}_{1}\right) \varphi(A)$ is a compact set, so $\tau(A) \tau\left(\mathcal{A}_{1}\right) \tau(A)$ is a compact set contained in a $\mathrm{C}^{*}$-algebra consisting of compact operators (Lemma 2.3). The rest of the proof now follows from our selfadjoint arguments in [5, Theorem 2.2].

Corollary 2.5. The geometrically compact elements of an operator semisimple algebra $\mathcal{A}$ form a $\mathrm{C}^{*}$-algebra.

Proof. In light of Lemma 2.3 and Theorem 2.4, it suffices to show that $\tau^{-1}$ is a $*$-homomorphism when restricted to the set of compact operators in $\tau(\mathcal{A})$. However, $\tau^{-1}$ is an isometry and therefore it preserves selfadjoint projections. By the spectral theorem it preserves all selfadjoint compact operators and the conclusion follows.

A minor modification of Lemma 2.2 shows that the socle of an operator semisimple algebra coincides with the set of all operators that can be isometrically represented as finite rank operators. Therefore Theorem 2.4 identifies the socle of such an algebra as the set of all elements with finite geometric rank.

We are now able to give a criterion for when the unit ball of an operator semisimple algebra contains nonzero geometrically compact elements.

Theorem 2.6. If $\mathcal{A}$ is an operator semisimple algebra, then the following statements are equivalent:

(i) The unit ball of $\mathcal{A}$ has nontrivial compact faces.

(ii) The unit ball of $\mathcal{A}$ has nontrivial finite-dimensional faces.

(iii) $\mathcal{A}$ contains nonzero geometrically compact elements.

(iv) $\mathcal{A}$ contains a nonzero atom $P$, i.e., a nonzero selfadjoint projection $P \in \mathcal{A}$ such that $\operatorname{dim} P \mathcal{A} P<\infty$.

Proof. (ii) $\Rightarrow$ (i): trivial.

(i) $\Rightarrow$ (iii): this follows from Theorem 1.3.

(iii) $\Rightarrow$ (iv): let $\mathcal{A}$ contain a nonzero geometrically compact element $A$. By Corollary 2.5 the geometrically compact elements form a $\mathrm{C}^{*}$-subalgebra $\mathcal{J} \subseteq \mathcal{A}$ consisting of compact operators. Hence $\mathcal{J}$ contains an atom; since $\mathcal{J} \subseteq \mathcal{A}$ is an ideal, so does $\mathcal{A}$. 
(iv) $\Rightarrow$ (ii): we claim that

$$
\mathcal{F}(I-P)=\left\{(I-P)+X \mid X=P X P \in \mathcal{A}_{1}\right\}
$$

is a face. By a standard argument, it suffices to show that if $\mathcal{F}(I-P)$ contains the midpoint of a line segment in the unit ball of $\mathcal{A}$, then the endpoints of the line segment also lie in $\mathcal{F}(I-P)$. Take $A, B \in \mathcal{A}$ with $A=(I-P)+X$ and $X=P X P$, and assume that $\|A \pm B\| \leq 1$. We need to show that $A \pm B \in \mathcal{F}(I-P)$.

Indeed, arguing as in the proof of Proposition 1.4, we produce bounded operators $S$ and $T$ such that

$$
B=S\left(I-A^{*} A\right)^{\frac{1}{2}}=\left(I-A A^{*}\right)^{\frac{1}{2}} T .
$$

However, $A^{*} A=(I-P)+P X^{*} X P$ and so

$$
I-A^{*} A=P-P X^{*} X P=\left(I-A^{*} A\right) P .
$$

Therefore, $\left(I-A^{*} A\right)^{\frac{1}{2}}=\left(I-A^{*} A\right)^{\frac{1}{2}} P$ and so

$$
B P=S\left(I-A^{*} A\right)^{\frac{1}{2}} P=B .
$$

A similar argument shows that $\left(I-A A^{*}\right)^{\frac{1}{2}}=P\left(I-A A^{*}\right)^{\frac{1}{2}}$ and so $B=P B$. Hence $B=P B P$, as desired.

Every operator algebra that contains the compact operators acts irreducibly and is therefore operator semisimple. The existence of finite-dimensional faces is not an issue here since any such algebra contains atoms. A later result, Theorem 4.3, is relevant.

By the reduced atomic representation of a $\mathrm{C}^{*}$-algebra $\mathcal{A}$ we mean a representation $\tau=\bigoplus_{a \in \mathbb{A}} \tau_{a}$, where $\left\{\tau_{a}\right\}_{a \in \mathbb{A}}$ is a maximal family of pairwise inequivalent irreducible representations of $\mathcal{A}$. It is an old result in $\mathrm{C}^{*}$-algebra theory (see Proposition 13.10 .13 in [30]) that the reduced atomic representation satisfies the properties of Definition 2.1. Therefore all $\mathrm{C}^{*}$-algebras are operator semisimple and so Theorem 2.6 applies here. (This was implicit in our earlier work in [5].)

A norm closed subalgebra $\mathcal{A}$ of an $\mathrm{AF} \mathrm{C}^{*}$-algebra is a (strongly maximal) TAF algebra if and only if it is the $\operatorname{limit} \underline{\lim }\left(\mathcal{A}_{i}, \varphi_{i}\right)$ of a directed system

$$
\mathcal{A}_{1} \stackrel{\varphi_{1}}{\longrightarrow} \mathcal{A}_{2} \stackrel{\varphi_{2}}{\longrightarrow} \mathcal{A}_{3} \stackrel{\varphi_{3}}{\longrightarrow} \mathcal{A}_{4} \stackrel{\varphi_{4}}{\longrightarrow} \cdots
$$

where for each $i \geq 1$, the subalgebra $\mathcal{A}_{i}$ satisfies:

(i) $\mathcal{A}_{i}$ is a direct sum of upper triangular matrix algebras.

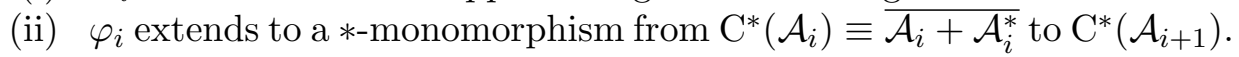

(iii) The extension of $\varphi_{i}$ maps matrix units to sums of matrix units.

We call (5) a presentation for the algebra; clearly it is not unique. TAF algebras have received much attention in recent years. A good reference for these and more general limit algebras is Power's monograph [44]. 
Two well-known examples of TAF algebras are the standard and refinement algebras. Define the standard embedding $\sigma_{k}$ by

$$
\sigma_{k}(A)=A \oplus A \oplus \cdots \oplus A \quad(k \text { factors })
$$

and the refinement embedding $\rho_{k}$ by

$$
\rho_{k}\left(\left[a_{s, t}\right]\right)=\left[a_{s, t} I_{k}\right],
$$

where $I_{k}$ is the $k \times k$ identity matrix. If all the embeddings $\varphi_{i}$ in the direct limit $\mathcal{A}=\underline{\lim }\left(\mathcal{A}_{i}, \varphi_{i}\right)$ are standard embeddings, $\mathcal{A}$ is said to be a standard algebra. If all the $\varphi_{i}$ are refinement embeddings, $\mathcal{A}$ is said to be a refinement algebra. An alternation algebra is a TAF algebra $\mathcal{A}=\underline{\lim }\left(\mathcal{A}_{i}, \varphi_{i}\right)$, where the $\varphi_{i}$ alternate between the standard and the refinement embeddings.

The embeddings $\varphi_{i}$ are said to be mixing if for every matrix unit $e_{s, t}^{(i)}$ we have $\varphi_{i}\left(e_{s, t}^{(i)}\right) \mathcal{A}_{i+1} \varphi_{i}\left(e_{s, t}^{(i)}\right) \neq 0$. The property of an embedding being mixing was introduced by Donsig in his study of semisimplicity [19] and was further exploited in [13]. It turns out that a TAF algebra $\mathcal{A}$ is semisimple if it admits a presentation $\mathcal{A}=\lim \left(\mathcal{A}_{i}, \varphi_{i}\right)$, where all the embeddings $\varphi_{i}$ are mixing. All standard embeddings are mixing, so the standard and alternation algebras are semisimple.

Let $\mathfrak{A}=\underline{\lim }\left(\mathfrak{A}_{i}, \varphi_{i}\right)$ be an AF $\mathrm{C}^{*}$-algebra and assume each $\mathfrak{A}_{i}$ decomposes as a direct $\operatorname{sum} \mathfrak{A}_{i}=\bigoplus_{j} \mathfrak{A}_{i j}$ of finite-dimensional full matrix algebras $\mathfrak{A}_{i j}$. A path $\Gamma$ for $\mathfrak{A}=\underline{\lim }\left(\mathfrak{A}_{i}, \varphi_{i}\right)$ is a sequence $\left\{\mathfrak{A}_{i j_{i}}\right\}_{i=1}^{\infty}$ such that for each pair of nodes $\left(\left(i, j_{i}\right),\left(i+1, j_{i+1}\right)\right)$ there exists an arrow in the Bratteli diagram for $\mathfrak{A}=\underline{\lim }\left(\mathfrak{A}_{i}, \varphi_{i}\right)$ joining them.

For each path $\Gamma$ consider a subsystem of the directed $\operatorname{limit} \underline{\lim }\left(\mathfrak{A}_{i}, \varphi_{i}\right)$ consisting of all the summands of $\mathfrak{A}$ that are never mapped into some $\mathfrak{A}_{i j_{i}} \in \Gamma$. Evidently this system is hereditary and directed upwards; therefore it determines an ideal $\mathcal{J}_{\Gamma}$ of $\mathfrak{A}$. The quotient $\mathfrak{A} / \mathcal{J}_{\Gamma}$ is the AF algebra corresponding to the remaining summands and the remaining embeddings. The summands that eventually get mapped into some $\mathfrak{A}_{i j_{i}} \in \Gamma$ are denoted by $\operatorname{summ}(\Gamma)$. Two paths $\Gamma=\left\{\mathfrak{A}_{i j_{i}}\right\}_{i=1}^{\infty}$ and $\Gamma^{\prime}=\left\{\mathfrak{A}_{i j_{i}^{\prime}}\right\}_{i=1}^{\infty}$ are said to be disjoint if for all but finitely many $i \in \mathbb{N}$, the nodes $\left(i j_{i}\right)$ and $\left(i j_{i}^{\prime}\right)$ are distinct.

Lemma 2.7. Let $\Gamma=\left\{\mathfrak{A}_{i j_{i}}\right\}_{i=1}^{\infty}$ and $\Gamma^{\prime}=\left\{\mathfrak{A}_{i j_{i}^{\prime}}\right\}_{i=1}^{\infty}$ be two disjoint paths for an $\mathrm{AF} \mathrm{C}^{*}$-algebra $\mathfrak{A}=\underline{\lim }\left(\mathfrak{A}_{i}, \varphi_{i}\right)$ and let $\omega=\underline{\lim } \omega_{i}$ and $\omega^{\prime}=\underline{\lim } \omega_{i}^{\prime}$ be pure states such that the $\omega_{i}$ and $\omega_{i}^{\prime}$ are supported on $\mathfrak{A}_{i j_{i}}$ and $\mathfrak{A}_{i j_{i}^{\prime}}$ respectively. Then the states $\omega$ and $\omega^{\prime}$ induce inequivalent GNS representations.

Proof. Assume that the states $\omega$ and $\omega^{\prime}$ produce equivalent irreducible representations $\pi$ and $\pi^{\prime}$. Then by [30, Theorem 10.2.6], there exists a unitary $u \in \mathfrak{A}$ such that $\omega=\omega^{\prime} \operatorname{ad}_{u}$, where $\operatorname{ad}_{u}(a)=u a u^{*}$. Every unitary in an AF algebra is a limit of unitaries in its finite-dimensional subalgebras. Hence there is a unitary $v$ in some $\mathfrak{B}_{i}$ such that $\left\|\omega-\omega^{\prime} \operatorname{ad}_{v}\right\|<1$. However the 
disjointness of the paths $\Gamma$ and $\Gamma^{\prime}$ shows that this cannot occur and the conclusion follows.

In $[\mathbf{1 3}]$ it was shown that the quotient $\mathcal{A} / \mathcal{J}$ of a TAF algebra $\mathcal{A}$ by a prime ideal $\mathcal{J}$ is operator primitive, i.e., it admits an isometric representation on a Hilbert space $\mathcal{H}$ such that the 2-ball of $\mathcal{A} / \mathcal{J}$ is weakly dense in the unit ball of $\mathcal{B}(\mathcal{H})$. In particular, a semisimple TAF subalgebra $\mathcal{A}$ of a primitive $\mathrm{C}^{*}$-algebra is operator primitive. We now generalize this to an arbitrary semisimple TAF algebra.

Theorem 2.8. A semisimple TAF algebra $\mathcal{A}=\underline{\lim }\left(\mathcal{A}_{i}, \varphi_{i}\right)$ is operator semisimple.

Proof. Before embarking on the proof we establish some terminology. If $e_{1}$ and $e_{2}$ are matrix units in $\mathcal{A}_{i_{1}}$ and $\mathcal{A}_{i_{2}}, i_{1}<i_{2}$, then we say that $e_{2}$ is a subordinate of $e_{1}$ if there exists a diagonal matrix unit $p \in \mathcal{A}_{i_{2}}$ such that $e_{2}=p e_{1}$. If $e_{2}$ and $f_{2}$ are subordinates of $e_{1}$ and $f_{1}$, then we say that $e_{2}$ majorizes $f_{2}$ if there exist matrix units $s, t \in \mathcal{A}_{i_{2}}$ such that $e_{2}=s f_{2} t$.

For the proof, start with any direct summand $\mathfrak{A}_{1, j_{1}}^{(1)}$ of $\mathfrak{A}_{1}$. Let $e_{1}^{(1)}$ be the characteristic vector of $\mathfrak{A}_{1, j_{1}}^{(1)}$, i.e., the vector at the top right corner of $\mathfrak{A}_{1, j_{1}}^{(1)}$. Since $\mathcal{A}$ is semisimple there exist a link $f_{2}^{(1)}$ for $e_{1}^{(1)}$ in some later summand of $\mathcal{A}$. For notational convenience we assume that $f_{2}^{(1)}$ belongs to some summand $\mathfrak{A}_{2, j_{2}}^{(1)}$ of $\mathfrak{A}_{2}$ and $\varphi_{1, j_{1}}^{(1)}$ is the mixing embedding from $\mathfrak{A}_{1, j_{1}}^{(1)}$ into $\mathfrak{A}_{2, j_{2}}^{(1)}$ that maps $e_{1}^{(1)}$ onto two copies, say $e_{(1,1)}^{(1)}$ and $e_{(1,2)}^{(1)}$, linked by $f_{2}^{(1)}$. Now let $e_{2}^{(1)}$ be the characteristic matrix unit of $\mathfrak{A}_{2, j_{2}}^{(1)}$ and let $f_{3}^{(1)}$ be a link for $e_{2}^{(1)}$ in $\mathfrak{A}_{3, j_{3}}^{(1)}$ and $\varphi_{2, j_{2}}^{(1)}$ the corresponding linking mapping. This way we construct a path $\Gamma^{(1)}$ for $\underset{\longrightarrow}{\lim }\left(\mathfrak{A}_{i}, \varphi_{i}\right)$. If $\operatorname{summ}\left(\Gamma^{(1)}\right)$ contains all the summands for all the finite-dimensional algebras $\mathfrak{A}_{i}$ we stop. Otherwise we chose a direct summand of $\mathfrak{A}$ not in $\operatorname{summ}\left(\Gamma^{(1)}\right)$ and we repeat the process described earlier. This way we define inductively a sequence $\left\{\Gamma^{(a)}\right\}_{a \in \mathbb{N}}$ of paths such that all maps involved with the nodes of the path are mixing and the union

$$
\bigcup_{a \in \mathbb{N}} \operatorname{summ}\left(\Gamma^{(a)}\right)
$$

contains all direct summands of $\mathfrak{A}$.

Given any path $\Gamma^{(a)}$ defined above, we now construct a pure state $\omega_{a}$ as follows: start with any unit vector, i.e., normalized sum of diagonal matrix units, $\xi_{1}^{(a)}$ in the central projection determined by $\mathfrak{A}_{1, j_{1}}^{(a)}$. Let $\omega_{1}^{(a)}$ be the vector state on $\mathfrak{A}_{1}$ determined by $\xi_{1}^{(a)}$, i.e., $\omega_{1}^{(a)}(A)=\left\langle A \xi_{1}^{(a)}, \xi_{1}^{(a)}\right\rangle$. Notice that $\xi_{1}^{(a)}$, being a sum of diagonal matrix units, it is mapped by $\varphi_{1, j_{1}}^{(1)}$ onto several copies in $\mathfrak{A}_{2}$. Two of them, say $\zeta_{1}^{(a)}$ and $\zeta_{2}^{(a)}$, are majorized by $e_{(1,1)}^{(a)}$ 
and $e_{(1,2)}^{(a)}$, respectively. Let

$$
\xi_{2}^{(a)}=\frac{1}{\sqrt{2}}\left(\zeta_{1}^{(a)}+\zeta_{2}^{(a)}\right)
$$

and let $\omega_{2}^{(a)}$ be the vector state on $\mathfrak{A}_{2}$ determined by $\xi_{2}^{(a)}$. Consequently, find two copies $\zeta_{1}^{(a)}$ and $\zeta_{2}^{(a)}$ of the image of $\xi_{2}^{(a)}$ in $\mathfrak{A}_{3}$ subordinated by $e_{(2,1)}^{(a)}$ and $e_{(2,2)}^{(a)}$, etc. This way we construct inductively a sequence $\left\{\omega_{a}\right\}_{a \in \mathbb{N}}$ of vector states. Since the states $\omega_{a}$ are supported in single summands of $\mathfrak{A}$, they are pure states and hence their direct limit $\omega_{a}$ is also pure.

Let $\left(\tau_{a}, \mathcal{H}_{a}, g_{a}\right)$ be the GNS representation induced by $\omega_{a}, a \in \mathbb{N}$, i.e., $\omega_{a}(A)=\left\langle\tau_{a}(A) g_{a}, g_{a}\right\rangle, A \in \mathfrak{A}$. By Lemma 2.7 the representations $\tau_{a}$ are mutually inequivalent. Therefore, Corollary 10.3.9 in [30] shows that the representation $\tau=\bigoplus_{a \in \mathbb{N}} \tau_{a}$ maps $\mathfrak{A}$ onto a weakly dense subalgebra of $\bigoplus_{a \in \mathbb{N}} \mathcal{B}\left(\mathcal{H}_{a}\right)$. Since $\bigcup_{a \in \mathbb{N}} \operatorname{summ}\left(\Gamma^{(a)}\right)$ contains all the direct summands from $\mathfrak{A}$, an easy argument shows that $\tau$ is faithful and so isometric. Therefore, Kaplansky's Theorem shows that $\tau$ satisfy the requirements of Definition 2.1 for $\mathfrak{A}$. It only remains to show that $\tau$ satisfies the same requirements for $\mathcal{A}$.

For each $i \in \mathbb{N}$ we construct a contractive map $\Phi_{i}: \mathfrak{A}_{i} \rightarrow \mathcal{A}_{i+1}$ as follows: let $u$ be a matrix unit in $\mathfrak{A}_{i}$. If $u$ does not belong to any of the nodes $\mathfrak{A}_{i, j_{i}}^{(a)}$ associated with the paths $\Gamma^{(a)}$, we set $\Phi_{i}(u)=0$. If $u \in \mathfrak{A}_{i, j_{i}}^{(a)}$ for some $a \in \mathbb{N}$, we consider the subordinates $u_{1}$ and $u_{2}$ of $u$ majorized by $e_{(i, 1)}^{(a)}$ and $e_{(i, 2)}^{(a)}$ respectively. Define $\Phi_{i}(u)$ to be the matrix unit with initial projection the initial projection of $u_{2}$ and final projection that of $u_{1}$. Since the matrix units $e_{(i, 1)}^{(a)}$ and $e_{(i, 2)}^{(a)}$ are linked in $\mathcal{A}_{i+1}$, we have that $\Phi_{i}(u) \in \mathcal{A}_{i+1}$. Moreover,

$$
\omega_{a}\left(B\left(A-2 \Phi_{i}(A)\right) C\right)=0
$$

for any $A, B, C \in \mathfrak{A}_{i}$. The weak density of the 2-ball of $\tau(\mathcal{A})$ in $\bigoplus_{a \in \mathbb{N}} \mathcal{B}\left(\mathcal{H}_{a}\right)_{1}$ now follows from the above equation and the fact that the collection of all vectors of the form

$$
\bigoplus_{a \in \mathbb{N}} \tau_{a}\left(A_{a}\right) g_{a}, \quad A_{a} \in \bigcup_{i \in \mathbb{N}} \mathfrak{A}_{i}
$$

where all but finitely many $A_{a}$ equal 0 , is a dense subset of the space $\bigoplus_{a \in \mathbb{N}} \mathcal{H}_{a}$

The techniques of the previous theorem are also applicable to inductive limits of block upper triangular matrices with mixing embeddings, thus showing that such algebras are operator semisimple.

A function $f: \mathcal{X} \longrightarrow \mathbb{C}$ is said to be unitary if $|f(x)|=1$, for all $x \in \mathcal{X}$.

Theorem 2.9. Let $\mathcal{X}$ be a compact Hausdorff space and let $\mathcal{A} \subseteq \mathrm{C}(\mathcal{X})$ be a norm closed algebra of continuous functions containing the constant functions. If the unitary functions in $\mathcal{A}$ separate the points of $\mathcal{X}$, then $\mathcal{A}$ is an operator semisimple algebra. 
Proof. Let $\mathcal{X}=\left\{x_{i} \mid i \in \mathbb{I}\right\}$ and for each $i \in \mathbb{I}$, consider the one-dimensional representation $\left(\mathcal{H}_{i}, \tau_{i}, z_{i}\right)$ such that $f\left(x_{i}\right)=\left\langle\tau_{i}(f) z_{i}, z_{i}\right\rangle, f \in \mathcal{A}$. Clearly, the representation $\tau=\bigoplus_{i \in \mathbb{I}} \tau_{i}$ is the reduced atomic representation of $\mathrm{C}(\mathcal{X})$. Hence, the strong closure of $\tau(\mathrm{C}(\mathcal{X}))$ equals the algebra $\mathcal{D}$ of all diagonal matrices on $\bigoplus_{i \in \mathbb{I}} \mathcal{H}_{i}$, i.e., $\mathcal{D}=l^{\infty}(\mathbb{I})$. We are to show that the strong closure of $\tau(\mathcal{A})_{1}$ equals $\mathcal{D}_{1}$.

Claim. Given $x_{i_{1}}, x_{i_{2}}, \ldots x_{i_{n}} \in \mathcal{X}$ and a unimodular number $w$, there exists $F$ in the strong closure of $\tau(\mathcal{A})_{1}$ such that $\left\langle F z_{i_{1}}, z_{i_{1}}\right\rangle=w$ and $\left\langle F z_{i_{k}}, z_{i_{k}}\right\rangle=1$, $k=2,3, \ldots, n$.

In order to prove the claim we make use of Möbius maps; if $g \in \mathcal{A}$ has norm 1 and $m$ is a Möbius transformation, then the composite function $m \circ g$ also belongs to $\mathcal{A}_{1}$. For the proof, for each $2 \leq l \leq n$ we choose a unitary function $g_{l}$ that separates $x_{i_{1}}$ from $x_{i_{l}}$. We apply a Möbius map to each $g_{l}$ to get $g_{l}^{\prime}$ such that $g_{l}^{\prime}\left(x_{i_{1}}\right)=1$ and either $g_{l}^{\prime}\left(x_{i_{k}}\right)=1$ or $e^{i \pi \alpha_{k l}}$ with $\alpha_{k l} \in[1 / n, 2 / n]$. If $g=\prod_{l} g_{l}^{\prime}$ then $g\left(x_{i_{1}}\right)=1$ and $g\left(x_{i_{l}}\right)=e^{\pi i \alpha_{l}}$, where $\alpha_{l} \in[1 / n, 2-2 / n]$. Now another application of a Möbius map produces a function $f_{s} \in \mathcal{A}$ such that $f_{s}\left(x_{i_{1}}\right)=w$ and $\left|f_{s}\left(x_{i_{l}}\right)-1\right| \leq 1 / s, l=2, \ldots, n$. Any weak limit of $\left\{\tau\left(f_{s}\right)\right\}_{s \in \mathbb{N}}$ proves the claim.

Since the strong closure of $\tau(\mathcal{A})_{1}$ is closed under multiplication, repeated use of the claim shows that given $x_{i_{1}}, x_{i_{2}}, \ldots x_{i_{n}} \in \mathcal{X}$ and a unimodular $n$ tuple $\left(w_{1}, w_{2}, \ldots, w_{n}\right)$, there exists $F$ in the strong closure of $\tau(\mathcal{A})_{1}$ such that $\left\langle F z_{i_{j}}, z_{i_{j}}\right\rangle=w_{j}$. A convexity argument now shows the desired equality.

Corollary 2.10. The Hardy space $\mathrm{H}^{\infty}$ and the disc algebra $\mathrm{A}(\mathbb{D})$ are operator semisimple.

Proof. Both satisfy the requirements of Theorem 2.9 [24, page 174].

One of the pleasing features of the representations $\tau$ in the proofs of Theorems 2.8 and 2.9 is that they extend to a $*$-representation of their enveloping $\mathrm{C}^{*}$-algebra. (We coin the term $\mathrm{C}^{*}$-semisimple for an operator semisimple algebra admitting such a representation.) Using the fact that the spatial norm on the tensor product of two $\mathrm{C}^{*}$-algebras is minimal, one can easily conclude that the spatial tensor product of $\mathrm{C}^{*}$-semisimple algebras is also $\mathrm{C}^{*}$-semisimple. Hence tensor products between $\mathrm{C}^{*}$-algebras, semisimple TAF algebras, Douglas algebras and irreducible algebras are operator semisimple thus providing additional examples of such algebras.

A subalgebra $\mathcal{A}$ of a $\mathrm{C}^{*}$-algebra $\mathfrak{A}$ is said to be Dirichlet if it satisfies $\overline{\mathcal{A}+\mathcal{A}^{*}}=\mathfrak{A}$. The prototypical example of a Dirichlet algebra is the disc algebra, as a subalgebra of the continuous functions on the circle. The term originates from the theory of functions. It has also been studied in the context of nonselfadjoint operator algebras by Arveson [9], Muhly and Solel [35] and others. All strongly maximal TAF algebras are easily seen to be Dirichlet. 
Proposition 2.11. Suppose that $\mathcal{A}$ is a Dirichlet subalgebra of an infinitedimensional simple $\mathrm{C}^{*}$-algebra $\mathfrak{A}$. If $\mathcal{A}$ is operator semisimple, the unit ball of $\mathcal{A}$ does not have any nontrivial compact faces.

Proof. Assume that the unit ball of $\mathcal{A}$ has a nontrivial compact face. Then Theorem 2.6 shows that $\mathcal{A}$ contains a nonzero atom $P$. Hence,

$$
P \mathfrak{A} P=P\left(\overline{\mathcal{A}+\mathcal{A}^{*}}\right) P \subseteq \overline{P \mathcal{A} P}+\overline{(P \mathcal{A} P)^{*}}=\mathbb{C} P
$$

and so $\mathfrak{A}$ contains a nonzero atom. But then, Theorem 2.6 contradicts the simplicity of $\mathfrak{A}$.

Combining Proposition 2.11 with Theorem 2.8 we obtain that the unit ball of the familiar standard, alternation and $A(\mathbb{Q}, \nu)$ algebras contain no nontrivial compact faces. (We do not know if the same is true for the unit ball of the refinement algebra.) Proposition 2.11 also applies to semicrossed products $\mathrm{C}(\mathcal{X}) \times_{\alpha} \mathbb{Z}^{+}$corresponding to minimal actions $\alpha$ on a compact metric space $\mathcal{X}$. In $[\mathbf{1 4}]$ it is shown that such semicrossed products are operator primitive. Since these are Dirichlet subalgebras of simple $\mathrm{C}^{*}$-algebras their unit ball contains no nontrivial compact faces.

\section{Spectral obstructions for the existence of compact faces}

In this section we obtain noncommutative analogs of Corollary 1.7. A free semigroup algebra is the weakly closed algebra generated by $n$ isometries with orthogonal ranges. The central example for these algebras is the "noncommutative Toeplitz algebra" $\mathcal{L}_{n}$ generated by the left regular representation of the free semigroup on $n$ letters. The study of $\mathcal{L}_{n}$ was initiated by Popescu $[41,42,43]$ in the context of dilation theory. A detailed analysis of $\mathcal{L}_{n}$ is contained in the papers of Davidson and Pitts [16, 17], Kribbs [33] and Arias and Popescu [7, 8], which develop the analytic structure. Apart from being a good example, it turns out that $\mathcal{L}_{n}$ is also a model for free semigroup algebras: the Structure Theorem in [15] shows that every free semigroup algebra has a $2 \times 2$ lower triangular form where the first column is a slice of a von Neumann algebra and the 22 entry is an algebra isomorphic to $\mathcal{L}_{n}$. Special classes of free semigroup algebras are important in the classification of certain representations of the Cuntz-Toeplitz algebra [16] and the construction of wavelets [10]

A continuous factor representation of the Cuntz algebra induces a nonatomic free semigroup algebra. Moreover, $\mathcal{L}_{n}$ is also nonatomic. Our next result shows that the unit ball of such free semigroup algebras contains no nontrivial geometrically compact elements. Specifically:

Theorem 3.1. If $\mathcal{A}$ is a free semigroup algebra, the following statements are equivalent:

(i) The unit ball of $\mathcal{A}$ has nontrivial compact faces. 
(ii) The unit ball of $\mathcal{A}$ has nontrivial finite-dimensional faces.

(iii) $\mathcal{A}$ contains nonzero geometrically compact elements.

(iv) $\mathcal{A}$ contains an atom.

Proof. As in the proof of Theorem 2.6, we only need to verify that (iii) implies (iv). Let $\mathcal{A}$ be a free semigroup algebra with no atoms. By Theorem 2.6 in $[\mathbf{1 5}], \mathcal{A}$ decomposes via a projection $P$ in $\mathcal{A}$ as $\mathcal{A}=\mathcal{M} P+P^{\perp} \mathcal{A} P^{\perp}$, where $\mathcal{M}$ is the von Neumann algebra generated by $\mathcal{A}$ and $\left.P^{\perp} \mathcal{A}\right|_{P^{\perp} \mathcal{H}}$ is isomorphic to $\mathcal{L}_{n}$. Let $\pi$ be the isomorphism from $\left.P^{\perp} \mathcal{A}\right|_{P^{\perp} \mathcal{H}}$ onto $\mathcal{L}_{n}$.

By way of contradiction assume that $A$ is a nontrivial geometrically compact element of $\mathcal{A}$. Then Proposition 1.4 shows that

$$
\left(A \mathcal{B}(\mathcal{H})_{1 / 2} A\right) \cap \mathcal{A} \subseteq \operatorname{cp}(\{B\}) ;
$$

and so the norm closure of $A \mathcal{A}_{1} A$ is norm compact.

We now claim that $P^{\perp} A P^{\perp}=0$. Indeed, notice that

$$
P^{\perp} A P^{\perp}\left(P^{\perp} \mathcal{A} P^{\perp}\right)_{1} P^{\perp} A P^{\perp} \subseteq P^{\perp}\left(A \mathcal{A}_{1} A\right) P^{\perp}
$$

is norm compact. Therefore, by applying $\pi$, we obtain a nonzero element $\hat{A}:=\pi\left(P^{\perp} A P^{\perp}\right)$ of $\mathcal{L}_{n}$ such that the closure of $\hat{A}\left(\mathcal{L}_{n}\right)_{1} \hat{A}$ is norm compact. Then either $A=0$ or, arguing as in the proof of Theorem 1.6, we conclude that the spectrum of $\hat{A}$ is countable. However, [16, Corollary 1.8] asserts that the spectrum of every nonscalar element of $\mathcal{L}_{n}$ is connected and contains more than one point. Since $\mathcal{L}_{n}$ is infinite-dimensional, $\hat{A}=0$.

The claim above, combined with the first paragraph of the proof, shows that $A=A P$. Now let $I-Q$ be the span of all atoms in $\mathcal{M}$. It is well-known that $Q$ is a central projection in $\mathcal{M}$ and that $Q \mathcal{M} Q$ is nonatomic. Since $\mathcal{A}$ contains no atoms, $P \mathcal{M} P$ is nonatomic and so $P \subseteq Q$. Hence

$$
A \mathcal{M}_{1} A=A P \mathcal{M}_{1} A=A(Q \mathcal{M} Q)_{1} A .
$$

At the same time,

$$
A \mathcal{M} A=A \mathcal{M} A P \subseteq \mathcal{M} P \subseteq \mathcal{A} ;
$$

therefore, by (6), $A$ is a geometrically compact element of the nonatomic algebra $Q \mathcal{M} Q$. Hence Theorem 2.6 implies $A=0$, a contradiction.

In spite of Theorem 3.1, the unit ball of a free semigroup algebra $\mathcal{A}$ always contains nontrivial faces. Indeed, if $\mathcal{A}$ contains a slice of a von Neumann algebra, this follows from Theorem 2.6. So it suffices to consider only the case where $\mathcal{A}=\mathcal{L}_{n}$.

Proposition 3.2. The unit ball of $\mathcal{L}_{n}$ contains nontrivial faces.

Proof. It is enough to prove that the boundary of the unit ball of $\mathcal{L}_{n}$ contains composite points. Indeed, for any such point $A$, the face $\mathcal{F}(A)$ is not a singleton or else $A$ is an extreme point. 
The wandering subspaces for any of the left creation operators $L_{i}$ span the Fock space $\mathcal{H}$. We therefore define an $\mathrm{H}^{\infty}$ functional calculus for $L_{i}$, which is well-defined, linear and isometric, as follows: if $\varphi \in \mathrm{H}^{\infty}$ then $\varphi\left(L_{i}\right):=\lim p_{n}\left(L_{i}\right)$, where $\left\{p_{n}\right\}_{n \in \mathbb{N}}$ is any bounded sequence of polynomials converging to $\varphi$.

Let $\varphi$ be a composite point on the boundary of the unit ball of $\mathrm{H}^{\infty}$ (the existence of such a $\varphi$ follows from the description of the extreme points of $\mathrm{H}^{\infty}$ in [24]). Then $\varphi\left(L_{i}\right)$ is the desired composite point.

The lack of nontrivial compact faces for the unit ball of $\mathcal{L}_{n}$ is due to the fact that the spectrum of any nonscalar operator in $\mathcal{L}_{n}$ is infinite and connected. In [28], Hoover, Peters and Wogen study the spectral properties of algebras that contain no zero divisors. The spectrum of any operator in such an algebra $\mathcal{A}$ is necessarily connected. However, $\mathcal{A}$ may contain nonzero quasinilpotents, so the arguments in the proof of Theorem 3.1 do not apply in this generality. Nevertheless, the unit ball of such an algebra $\mathcal{A}$ does not contain any nontrivial finite-dimensional faces. Indeed, $\mathcal{A}$ does not contain any nonzero nilpotent operators, so no finite-dimensional subalgebras apart from the trivial one. The conclusion now follows from Theorem 1.8.

If $\mathcal{A}$ is a Banach algebra and $\alpha$ an automorphism of $\mathcal{A}$, the semicrossed product $\mathcal{A} \times{ }_{\alpha} \mathbb{Z}^{+}$is the enveloping Banach algebra of $l^{1}\left(\mathcal{A}, \mathbb{Z}^{+}, \alpha\right)$ with respect to the class of contractive Hilbert space representations. An interesting feature of the class of algebras studied by Hoover, Peters and Wogen in [28] is that it is closed under semicrossed products by $\mathbb{Z}^{+}$. (If $\mathcal{A} \times{ }_{\alpha} \mathbb{Z}^{+}$contains $x$ and $y$ such that $x y=0$, then the lowest Fourier coefficients of $x$ and $y$ are necessarily zero divisors in $\mathcal{A}$.) Hence:

Theorem 3.3. Let $\mathcal{A}$ be an algebra that contains no zero divisors and let $\alpha$ be an automorphism of $\mathcal{A}$. Then the unit ball of $\mathcal{A} \times{ }_{\alpha} \mathbb{Z}^{+}$does not contain any nontrivial finite-dimensional faces.

The theorem above applies in particular to the algebras $\mathrm{A}(\mathbb{D}) \times{ }_{\alpha} \mathbb{Z}^{+}$and $\mathrm{H}^{\infty} \times_{\alpha} \mathbb{Z}^{+}$, studied in $[\mathbf{2 7}]$.

Notice that $\mathrm{C}(\mathcal{X}), \mathcal{X}$ compact metric space, contains zero divisors and so Theorem 3.3 does not apply in this case. Crossed products of the form $\mathrm{C}(\mathcal{X}) \times_{\alpha} \mathbb{Z}^{+}$were studied in the previous section with the use of representation theory. They can also be studied with the use of Corollary 1.10 as follows:

Theorem 3.4. Let $\mathcal{X}$ be a compact connected metric space and let $\varphi$ be a homeomorphism of $\mathcal{X}$ with a dense set of recurrent points. Then the unit ball of $\mathrm{C}(\mathcal{X}) \times{ }_{\varphi} \mathbb{Z}^{+}$has no finite-dimensional faces.

Proof. By [20], the algebra $\mathrm{C}(\mathcal{X}) \times_{\varphi} \mathbb{Z}^{+}$is semisimple. We claim that $\mathrm{C}(\mathcal{X}) \times{ }_{\varphi} \mathbb{Z}^{+}$contains no nontrivial idempotents. 
Indeed, let $Q \in \mathrm{C}(\mathcal{X}) \times{ }_{\varphi} \mathbb{Z}^{+}$be an idempotent and let $Q=\sum_{i=0}^{\infty} f_{i} t^{i}$, $f_{i} \in \mathrm{C}(\mathcal{X})$ be its Fourier expansion. Since $Q$ is an idempotent, $f_{0}$ is an idempotent. But $\mathcal{X}$ is connected and so $f_{0}$ is either 0 or $I$.

If $f_{0}=0$ then the lowest Fourier coefficient in the expansion of $Q^{2}$ is of order 2 and so $f_{1}=0$. Consequently, the lowest Fourier coefficient in the expansion of $Q^{2}$ is of order 4 , so $f_{2}=f_{3}=0$. Repeated applications of this argument show that $f_{n}=0, n \geq 0$ and so $Q=0$.

If $f_{0}=I$ then the second Fourier coefficient of $Q^{2}$ is $2 f_{1}$. Hence $f_{1}=0$. Repeated applications of this argument show that the rest of the Fourier coefficients are equal to 0 and so $Q=I$ in this case. This proves the claim.

Since $\mathrm{C}(\mathcal{X}) \times_{\varphi} \mathbb{Z}^{+}$contains no nontrivial idempotents, the conclusion follows from Corollary 1.10.

\section{Concluding remarks}

The operator semisimple and $\mathrm{C}^{*}$-semisimple algebras can be thought as special cases of diagonal algebras. An operator algebra $\mathcal{A}$ is said to be block diagonalizable if there exists a bicontinuous (but not necessarily isometric) representation $\tau$, satisfying the rest of the properties in Definition 2.1. Several results of Section 2, such as Lemmas 2.2, 2.3 and one direction in Theorem 2.4, are valid in this more general context.

By the Wedderburn-Artin Theorem, all finite-dimensional semisimple algebras are block diagonalizable. However, there are semisimple operator algebras that fail that property, as the following example shows:

Example 4.1. A semisimple operator algebra that is not block diagonalizable.

Let $\mathcal{L}=\{0, M, N, I\}$, where $M, N$ are closed subspaces satisfying

$$
M \cap N=M^{\perp} \cap N^{\perp}=0,
$$

so that the sum $M+N$ is not closed. Let $\mathcal{A}=\operatorname{Alg} \mathcal{L}$ be the algebra of all operators leaving both $M$ and $N$ invariant. By an old result of Longstaff, a rank one operator $A \in \mathcal{A}$ is of the form $R=e \otimes f$, where either $e \in M^{\perp}$ and $f \in N$ or $e \in N^{\perp}$ and $f \in M$. The rank one subalgebra $\mathcal{R}(\mathcal{L})$ of $\operatorname{Alg} \mathcal{L}$ consists of all sums of rank one operators in $\operatorname{Alg} \mathcal{L}$. In [39] it is shown that $\mathcal{R}(\mathcal{L})$ is weakly dense in $\operatorname{Alg} \mathcal{L}$ (rank one density).

The semisimplicity of $\mathcal{A}$ follows from [31]. We are to show that $\mathcal{A}$ is not block diagonalizable.

By way of contradiction assume that $\left(\tau_{a}, \mathcal{H}_{a}\right), a \in \mathbb{A}$, is the family of representations of $\mathcal{A}$ implementing the operator semisimplicity and let $\tau=$ $\bigoplus_{a \in \mathbb{A}} \tau_{a}$. Notice that $R \mathcal{A} R$ is one-dimensional, for any rank one operator $R \in \mathcal{A}$, and so $\tau(R)$ is also a rank one operator. Since $\mathcal{R}(\mathcal{L})$ is dense in $\operatorname{Alg} \mathcal{L}$, $\tau(\mathcal{A})$ contains the set $\mathcal{K}$ of all compact operators in the diagonal algebra 
$\bigoplus_{a \in \mathbb{A}} \mathcal{B}\left(\mathcal{H}_{a}\right)$. The restriction of $\tau^{-1}$ on $\mathcal{K}$ is similar to a $*$-representation. Therefore there exists an invertible operator $S$ such that

$$
\mathcal{R}(S \mathcal{L}) \subseteq S \tau^{-1}(\mathcal{K}) S^{-1} \subseteq \operatorname{Alg} S \mathcal{L} .
$$

However, $S \tau^{-1}(\mathcal{K}) S^{-1}$ is selfadjoint and so the density of $\mathcal{R}(S \mathcal{L})$ in $\operatorname{Alg} S \mathcal{L}$ implies that $\operatorname{Alg} S \mathcal{L}$ is selfadjoint. Hence $S \mathcal{L}$ is orthocomplemented and so the sum $M+N$ is closed, a contradiction.

In this paper we addressed the problem of existence for nontrivial finitedimensional faces. The problem of characterizing which operators belong to such faces is much harder. Indeed, by the Krein-Milman Theorem such faces are the closed convex hull of their extreme points and therefore one needs to have a good understanding of the extreme points for the unit ball. This is also corroborated by the following:

Proposition 4.2. Let $\mathcal{X}$ be a normed space and let $x$ be an element of its unit ball. If $x$ belongs to a finite-dimensional face, there exists an extreme point $e$ for the unit ball of $\mathcal{X}$ and an element $f$ with finite geometric rank such that $x=e+\lambda f$, for some $\lambda \in \mathbb{R}$.

Proof. Since $\mathcal{F}$ is finite-dimensional, $\mathcal{F}(x)$ is also finite-dimensional and by the Krein-Milman Theorem it contains an extreme point $e$. Moreover there exists an element $f$ in the affine hull of $\mathcal{F}(x)$ such that $x=e+f^{\prime}$. By Lemma 1.2, the affine hull of $\mathcal{F}(x)$ equals $[\operatorname{cp}(\{x\})]$. Since $\operatorname{cp}(\{x\})$ is convex, a moment's reflection shows that $[\operatorname{cp}(\{x\})]$ consists of multiples of $\operatorname{cp}(\{x\})$ and so there exists an element $f \in \operatorname{cp}(\{x\})$ such that $f^{\prime}=\lambda f$, for some $\lambda \in \mathbb{R}$. However,

$$
\mathrm{cp}^{(2)}(\{f\}) \subseteq \mathrm{cp}^{(3)}(\{x\})=\mathrm{cp}(\{x\}),
$$

which shows that $f$ has finite geometric rank, as desired.

Even though we have a complete understanding of the elements with finite geometric rank, we are far from characterizing the extreme points for operator semisimple algebras. For instance, the problem of characterizing the extreme points of the standard algebra is open [26]. Also compare the nature of extreme points in the unit ball of a $\mathrm{C}^{*}$-algebra with that of $\mathrm{H}^{\infty}$ [24, page 138]. However, the situation in operator primitive algebras is much better.

Theorem 4.3. Let $\mathcal{A}$ be an operator algebra containing the compact operators and let $A \in \mathcal{A}$ with $\|A\|=1$. Then the operator $A$ belongs to a finite-dimensional face of $\mathcal{A}_{1}$ if and only if

$$
\operatorname{dim}\left(\left(I-A A^{*}\right) \mathcal{A}\left(I-A^{*} A\right)\right)<\infty .
$$

Proof. Assume first that $\operatorname{dim}\left(\left(I-A A^{*}\right) \mathcal{A}\left(I-A^{*} A\right)\right)<\infty$, so both $I-A A^{*}$ and $I-A^{*} A$ are finite rank operators. Hence if $P$ and $Q$ are the range 
projections of $\left(I-A^{*} A\right)^{1 / 2}$ and $\left(I-A A^{*}\right)^{1 / 2}$ respectively, then $P \mathcal{A} Q \subseteq \mathcal{A}$ is finite-dimensional.

Let $B \in \operatorname{cp}(\{A\})$. Then [34, Lemma 1] shows that there exist bounded operators $S$ and $T$ such that

$$
B=S\left(I-A^{*} A\right)^{1 / 2}=\left(I-A A^{*}\right)^{1 / 2} T
$$

and so $B=Q B P$. Therefore $\operatorname{cp}(\{A\})$ is a finite-dimensional, and Lemma 1.2 shows that $A$ belongs to the finite-dimensional face $\mathcal{F}(A)$.

Conversely, assume that $\mathcal{F}(A)$ is finite-dimensional face and so, by Theorem 1.8, $\mathrm{S}(\mathcal{F}(A))=\operatorname{cp}(\{A\})$ is a finite-dimensional hereditary subalgebra of $\mathcal{A}$. A moment's reflection shows that there exist finite-dimensional projections $P, Q$ such that $\operatorname{cp}(\{A\})=P \mathcal{A} Q$.

We now claim that

$$
(I-P) \cap\left(I-A^{*} A\right)^{1 / 2}(\mathcal{H})=(I-Q) \cap\left(I-A A^{*}\right)^{1 / 2}(\mathcal{H})=\{0\} .
$$

Indeed, by way of contradiction assume that one of the above intersections, say the first one, is nontrivial. Consider a unit vector $\left(I-A A^{*}\right)^{1 / 2} f \in I-P$ and let $e$ be a vector of norm $1 / 2$ such that $\left(I-A^{*} A\right)^{1 / 2} e \neq 0$. Then $[34$, Corollary 1] shows that the rank one operator

$$
R=\left(I-A^{*} A\right)^{1 / 2} e \otimes\left(I-A A^{*}\right)^{1 / 2} f=\left(I-A A^{*}\right)^{1 / 2}(e \otimes f)\left(I-A^{*} A\right)^{1 / 2}
$$

belongs to $\operatorname{cp}(\{A\})$ and so our earlier observations show that $R=P R Q$, a contradiction that proves the claim.

From the claim above it follows that

$$
(I-P) \cap\left(I-A^{*} A\right)(\mathcal{H})=(I-Q) \cap\left(I-A A^{*}\right)(\mathcal{H})=\{0\} .
$$

Since both $I-P$ and $I-Q$ are of finite codimension, we conclude that the ranges of both $I-A^{*} A$ and $I-A A^{*}$ are finite-dimensional, as promised.

Arguments similar to the ones above lead to a generalization of Kadison's characterization of the extreme points of the unit ball of a $\mathrm{C}^{*}$-algebra [29]. Indeed one can show that a contraction $A$ belongs to a finite-dimensional face of a $\mathrm{C}^{*}$-algebra $\mathcal{A}$ if and only if $\operatorname{dim}\left(\left(I-A A^{*}\right) \mathcal{A}\left(I-A^{*} A\right)\right)<\infty$. (This was first shown to us by Anoussis with a different proof.)

Another example that illustrates the complexities associated with extreme points in nonselfadjoint operator algebras is the following: let $\mathcal{A}$ be an operator algebra acting on a Hilbert space $\mathcal{H}$ and consider the operator algebra

$$
\mathcal{T}(\mathcal{A})=\left\{\left(\begin{array}{ll}
\lambda & 0 \\
f & A
\end{array}\right) \mid A \in \mathcal{A}, f \in \mathcal{H}, \lambda \in \mathbb{C}\right\}
$$

with the obvious multiplications.

Proposition 4.4. The rank one operator $R=(1,0) \otimes(0, f)$ is an extreme point for the unit ball of $\mathcal{T}(\mathcal{A})$ if and only if $f$ is a separating unit vector for $\mathcal{A}^{*}$. 
Proof. First assume $f$ is a separating unit vector for $\mathcal{A}^{*}$, and let $X \in \mathcal{T}(\mathcal{A})$ be such that $\|R \pm X\|=1$. Then

$$
X^{*} X \leq I-(1,0) \otimes(1,0)
$$

and

$$
X X^{*} \leq I-(0, f) \otimes(0, f) .
$$

The first inequality shows that $\mathcal{X} \in \mathcal{A}$. The second one shows that the range of $\mathcal{X}$ is orthogonal to $f$ and so $\langle X g, f\rangle=0, \forall g \in \mathcal{H}$. Hence, $\left\langle g, X^{*} f\right\rangle=0$, $\forall g \in \mathcal{H}$, and so $X^{*} f=0$. Since $f$ is separating for $\mathcal{A}^{*}, X=0$ and so $R$ is an extreme point.

Reversing the arguments above we obtain the other direction in the Theorem.

The identification of separating vectors for an operator algebra $\mathcal{A}$, i.e., the cyclic vectors for $\mathcal{A}^{\prime}$, is an important and difficult problem. Most notable is the work in [21] for the adjoint of the unilateral shift.

Note that the algebras of the form $\mathcal{T}(\mathcal{A})$, when $\mathcal{A}^{*}$ admits separating vectors, show that Theorem 2.4 fails for algebras that are not operator semisimple. Indeed, by way of contradiction assume that an operator $T \in \mathcal{T}(\mathcal{A})$ is geometrically compact if and only if there exists an isometric representation $\varphi$ of $\mathcal{T}(\mathcal{A})$ such that $\varphi(T)$ is a compact operator. This applies in particular to any operator of the form $T=(e, 0) \otimes(0, f)$ and so such an operator is geometrically compact. This contradicts however Proposition 4.4.

Acknowledgements. The author has benefited from many discussions with Mihalis Anoussis. He also acknowledges the assistance of Ken Davidson in the proof of Theorem 2.9.

\section{References}

[1] C. Akemann and J. Anderson, Lyapunov theorems for operator algebras, Mem. Amer. Math. Soc., 94(458) (1991), MR 1086563 (92e:46113), Zbl 0769.46036.

[2] C.A. Akemann and G.K. Pedersen, Facial structure in operator algebra theory, Proc. London Math. Soc., 64 (1992), 418-448, MR 1143231 (93c:46106), Zbl 0759.46050.

[3] J.C. Alexander, Compact Banach algebras, Proc. London Math. Soc., 18 (1968), 1-18, MR 0229040 (37 \#4618), Zbl 0184.16502.

[4] E. Alfsen and E. Effros, Structure in real Banach spaces I, II, Ann. of Math., 96 (1972), 98-128; ibid., 96 (1972), 129-173, MR 0352946 (50 \#5432), Zbl 0248.46019.

[5] M. Anoussis and E. Katsoulis, Compact operators and the geometric structure of $\mathrm{C}^{*}$ algebras, Proc. Amer. Math. Soc., 124 (1996), 2115-2122, MR 1322911 (96i:46068), Zbl 0857.46034.

[6] M. Anoussis and E. Katsoulis, Compact operators and the geometric structure of nest algebras, Indiana U. Math. J., 45 (1996), 1175-1191, MR 1444482 (98e:47066a), Zbl 0883.47036. 
[7] A. Arias and G. Popescu, Factorization and reflexivity on Fock spaces, Integral Equations Operator Theory, 23 (1995), 268-286, MR 1356335 (97e:47066), Zbl 0842.47026.

[8] A. Arias and G. Popescu, Noncommutative interpolation and Poisson transforms, Israel J. Math., 115 (2000), 205-234, MR 1749679 (2001i:47021), Zbl 0967.47045.

[9] W. Arveson, Analyticity in operator algebras, Amer. J. Math., 89 (1967), 578-642, MR 0223899 (36 \#6946), Zbl 0183.42501.

[10] O. Bratteli and P. Jorgensen, Iterated function systems and permutation representations of the Cuntz algebra, Mem. Amer. Math. Soc., 139(663), 1999, MR 1469149 (99k:46094a), Zbl 0935.46057.

[11] J. Conway, A Course in Functional Analysis (2nd ed.), Graduate Texts in Mathematics, 96, Springer-Verlag, 1990, MR 1070713 (91e:46001), Zbl 0706.46003.

[12] K. Davidson, $\mathrm{C}^{*}$-Algebras by Example, Fields Institute Monographs, 6, American Mathematical Society, 1996, MR 1402012 (97i:46095), Zbl 0958.46029.

[13] K. Davidson and E. Katsoulis, Primitive limit algebras and $\mathrm{C}^{*}$-envelopes, Adv. Math., 170(2) (2002), 181-205, MR 1932328 (2003h:47140), Zbl 1011.46048.

[14] K. Davidson and E. Katsoulis, work in progress.

[15] K. Davidson, E. Katsoulis and D. Pitts, The structure of free semigroup algebras, J. Reine Angew. Math., 533 (2001), 99-125, MR 1823866 (2002a:47107), Zbl 0967.47047.

[16] K. Davidson and D. Pitts, Invariant subspaces and hyper-reflexivity for free semigroup algebras, Proc. London Math. Soc., 78 (1999), 401-430, MR 1665248 (2000k:47005), Zbl 0997.46042.

[17] K. Davidson and D. Pitts, The algebraic structure of non-commutative analytic Toeplitz algebras, Math. Ann., 311 (1998), 275-303, MR 1625750 (2001c:47082), Zbl 0939.47060.

[18] K. Davidson and D. Pitts, Nevanlinna-Pick interpolation for non-commutative analytic Toeplitz algebras, Integral Equations Operator Theory, 31 (1998), 321-337, MR 1627901 (2000g:47016), Zbl 0917.47017.

[19] A.P. Donsig, Semisimple triangular AF algebras, J. Funct. Anal., 111 (1993), 323349, MR 1203457 (94b:46084), Zbl 0808.47031.

[20] A.P. Donsig, A. Katavolos and A. Manoussos, The Jacobson radical for analytic crossed products, J. Funct. Anal., 187 (2001), 129-145, MR 1867344 (2002k:46170), Zbl 0998.46034.

[21] R.G. Douglas, H.S. Shapiro and A.L. Shields, Cyclic vectors and invariant subspaces for the backward shift operator, Ann. Inst. Fourier (Grenoble), 20 (1970), 37-76, MR 0270196 (42 \#5088), Zbl 0186.45302.

[22] C. Edwards and G. Ruttimann, On the facial structure of the unit balls in a GL-space and its dual, Math. Proc. Cambridge Philos. Soc., 98 (1985), 305-322, MR 0795896 (87a:46114), Zbl 0577.46007.

[23] C. Edwards and G. Ruttimann, On the facial structure of the unit balls in a $\mathrm{JBW}^{*}$ triple and its predual, J. London Math. Soc., 38 (1988), 317-332, MR 0966303 (90b:46129), Zbl 0621.46043.

[24] K. Hoffman, Banach Spaces of Analytic Functions, Dover Publications Inc., 1988, MR 1102893 (92d:46066), Zbl 0734.46033.

[25] T. Hudson and E. Katsoulis, Primitive triangular UHF algebras, J. Funct. Anal., 160 (1998), 1-27, MR 1658724 (99m:46135), Zbl 0923.46053. 
[26] T. Hudson, E. Katsoulis and D. Larson, Extreme points in triangular UHF algebras, Trans. Amer. Math. Soc., 349 (1997), 3391-3400, MR 1407493 (97m:47058), Zbl 0883.47035.

[27] T. Hoover, Isomorphic operator algebras and conjugate inner functions, Michigan Math. J., 39 (1992), 229-237, MR 1162033 (93h:47056), Zbl 0771.47013.

[28] T. Hoover, J. Peters and W. Wogen, Spectral properties of semicrossed products, Houston J. Math., 19 (1993), 649-660, MR 1251615 (94k:47067), Zbl 0795.46053.

[29] R. Kadison, Isometries of operator algebras, Ann. of Math., 54 (1951), 325-338, MR 0043392 (13,256a), Zbl 0045.06201.

[30] R.V. Kadison and J.R. Ringrose, Fundamentals of the theory of operator algebras, Vol. II, Academic Press, London, 1983, MR 0859186 (88d:46106), Zbl 0601.46054.

[31] A. Katavolos and E. Katsoulis, Semisimplicity in operator algebras and subspace lattices, J. London Math. Soc., 42 (1990), 365-372, MR 1083452 (92b:47066), Zbl 0739.47019.

[32] T. Kato, Notes on some inequalities for linear operators, Math. Ann., 125 (1952), 208-212, MR 0053390 (14,766e), Zbl 0048.35301.

[33] D.W. Kribs, Factoring in non-commutative analytic Toeplitz algebras, J. Operator Theory, 45(1) (2001), 175-193, MR 1823067 (2002g:47151), Zbl 0996.47065.

[34] R. Moore and T. Trent, Extreme points of certain operator algebras, Indiana U. Math. J., 36 (1987), 645-650, MR 0905616 (89d:47103), Zbl 0653.47023.

[35] P. Muhly and B. Solel, Dilations for representations of triangular algebras Bull. London Math. Soc., 21 (1989), 489-495, MR 1005829 (90i:47045), Zbl 0721.46034.

[36] G.J. Murphy, C*-Algebras and Operator Theory, Academic Press, Inc., Boston, MA, 1990, MR 1074574 (91m:46084), Zbl 0714.46041.

[37] J. Orr and J. Peters, Some representations of TAF algebras, Pacific. J. Math., 167 (1995), 129-161, MR 1318167 (96c:46055), Zbl 0822.46066.

[38] T. Palmer, Banach Algebras and the General Theory of *-Algebras, I: Algebras and Banach Algebras, Encyclopedia of Mathematics and its applications, 49, Cambridge University Press, 1994, MR 1270014 (95c:46002), Zbl 0809.46052.

[39] M. Papadakis, On hyperreflexivity and rank one density for non-CSL algebras, Studia Math., 98 (1991), 11-17, MR 1110095 (92g:47062), Zbl 0755.47030.

[40] G. Pedersen, $\mathrm{C}^{*}$-Algebras and their Automorphism Groups, London Mathematical Society Monographs, 14, Academic Press Inc. London-New York, 1979, MR 0548006 (81e:46037), Zbl 0416.46043.

[41] G. Popescu, Characteristic functions for infinite sequences of noncommuting operators, J. Operator Theory, 22 (1989), 51-71, MR 1026074 (91m:47012), Zbl 0703.47009.

[42] G. Popescu, Multi-analytic operators and some factorization theorems, Indiana Univ. Math. J., 38 (1989), 693-710, MR 1017331 (90k:47019), Zbl 0661.47020.

[43] G. Popescu, Multi-analytic operators on Fock spaces, Math. Ann., 303 (1995), 31-46, MR 1348353 (96k:47049), Zbl 0835.47015.

[44] S.C. Power, Limit Algebras: An Introduction to Subalgebras of $C^{*}$-Algebras, Pitman Research Notes in Math., 278, Longman, London, 1993, MR 1204657 (94g:46001), Zbl 0917.46001. 
[45] M. Rørdam, Advances in the theory of unitary rank and regular approximation, Ann. of Math., 128 (1988), 153-172, MR 0951510 (90c:46072), Zbl 0659.46052.

[46] J. Rovnyak, Operator-valued analytic functions of constant norm, Czechoslovak Math. J., 39 (1989), 165-168, MR 0983493 (90f:47019), Zbl 0686.47013.

[47] K. Ylinen, A note on the compact elements of $C^{*}$-algebras, Proc. Amer. Math. Soc., 35 (1972), 305-306, MR 0296716 (45 \#5775), Zbl 0257.46085.

Received June 3, 2002 and revised November 19, 2003. This research was partially supported by a grant from ECU.

Department of Mathematics

East Carolina University

GreENVILLE NC 27858

E-mail address: KatsoulisE@mail.ecu.edu 